\title{
Discovering Remote Software Services that Satisfy Requirements: Patterns for Query Reformulation
}

\author{
Nektarios Dourdas ${ }^{1}$, Xiaohong Zhu ${ }^{2}$, Neil Maiden ${ }^{2}$, Sara Jones ${ }^{2}$, \\ and Konstantinos Zachos ${ }^{2}$ \\ ${ }^{1}$ SAP, Walldorf, Germany \\ ${ }^{2}$ Centre for HCI Design, City University, UK \\ NDourdas@gmail.com, XZhu@soi.city.ac.uk, \\ N.A.M.Maiden@city.ac.uk, S.V.Jones@soi.city.ac.uk, \\ KZachos@soi.city.ac.uk
}

\begin{abstract}
Developing service-centric applications will require developers to discover candidate services during requirements processes. However such discovery is challenging due to the ontological mismatch between requirement and service descriptions. We propose patterns to re-express requirements-based service queries using classes of solution service, to increase the likelihood of discovering relevant services from service registries. We report a prototype pattern language developed for service-based vehicle fleet management, and demonstrate its use with an example.
\end{abstract}

\section{Introduction}

Recent developments in web services and standards have been rapid. Standards such as SOAP and WSDL are well established. Major vendors such as IBM, Microsoft, Sun and HP provide support for services in their development platforms, and many companies are offering web service interfaces to their systems. UDDI offers established directories of service providers. Leavitt [2004] reports that worldwide spending on web services software projects will reach $\$ 11$ billion by 2008 , compared to $\$ 1.1$ billion in 2003. Given these trends, development of service-centric systems with software services available over the internet is a new research challenge for software engineering - a challenge that we are addressing in the EU-funded SeCSE Integrated Project (secse.eng.it).

Developing service-centric systems has important consequences for how developers determine the requirements of these systems. We conjecture that developers will want to discover candidate services early in the development process, to explore what capabilities are possible, design an architecture compliant with these capabilities, and trade-off quality-of-service requirements [Jones et al. 2005]. To do this, requirements must form elements of service queries with which to discover candidate services for a new application [Schmid et al. 2005].

However, successfully discovering software services using requirements poses research challenges. Requirements express desirable properties of the problem domain 
[Jackson 1995] in terms of the goals of the service consumer, for example repair a fault in a car engine. In contrast, service specifications describe solution behaviour what the piece of software does, largely independent of the problem domain, to maximise reuse of the software within and across domains. For example, services to repair an engine fault include collect and diagnose engine data, and locate garages equipped to undertake the repair. SeCSE's current service discovery environment implements an algorithm for discovering services from requirements specifications using query expansion and word sense disambiguation techniques [Zachos \& Maiden 2005] to handle the problem of incomplete requirements.

Alas query expansion alone cannot resolve the mismatch that arises because the problem query and solution service are inevitably expressed using different ontologies. To overcome this ontological mismatch, we are extending the SeCSE algorithm with patterns that encapsulate knowledge about classes of proven service solutions to classes of requirement problems. Knowledge about how service solution classes can solve classes of problem can be applied to change the ontology of the service query to the solution domain, thus increasing the likelihood of discovering services that are compliant with consumer requirements. In the above simple example, the resulting service query will seek services that collect and diagnose engine data and locate garages, rather than simply repair an engine.

In SeCSE we are developing pattern languages in chosen automotive domains. One domain that we have developed a pattern language for is vehicle fleet management in DaimlerChrysler. DaimlerChrysler envisage that company managers of Mercedes car fleets will use remote software services with on-board systems to manage cars that are distributed across the fleet. This paper describes how the pattern language was developed and represented, and demonstrates its usefulness in service discovery using an example from DaimlerChrysler.

The remainder of this paper is in 4 sections. Section 2 describes SeCSE's patternbased approach to service discovery. Section 3 describes the pattern language for vehicle fleet management elicited from DaimlerChrysler engineers. Section 4 uses an example to demonstrate potential benefits from patterns-based service discovery. The last section describes how we are implementing pattern-based discovery, and outlines future research.

\section{Service-Centric Systems and Patterns}

Service-centric systems are systems that integrate services from different providers regardless of the underlying operating systems or programming languages of those applications. A software service is a set of operations offered by an application. Users access services through well-defined interfaces independently of where the service is executed. Software services accessed over the internet are called web services. Automotive manufacturers such as DaimlerChrysler can benefit from service-centric applications because software and information used in these applications is not embedded and isolated in cars, but available and up-to-date on remote servers.

Patterns research started with the architect Christopher Alexander, who described over 250 architectural building patterns at different levels of abstraction 
[Alexander 1979]. Equivalent software patterns became popular in object-oriented design [Gamma et. al. 1995], and have been applied subsequently to many phases of software development. Our research uses Alexander's [1979] original definition of a pattern as a proven solution to a recurring problem in a context of use. In SeCSE we employ this definition to describe: (i) classes of problem that re-occur during the design of service-centric applications, and: (ii) classes of candidate services proven to solve these problems. Returning to the above example, the problem element of the pattern describes a class of problem in a context, i.e. a vehicle driving and developing a fault that needs analysis and repair. The solution element describes classes of service to collect engine fault data, analyze and diagnose this data, and send the data to a parts supplier. To decouple development of SeCSE patterns from the publication of concrete software services by service providers in heterogeneous service registries such as UDDI, the patterns do not reference concrete services in these registries. Instead, each pattern specifies descriptions of classes of service that an algorithm uses to transform the service query and discover instances of new software services in the registries.

\subsection{Previous Work}

There has been little research into patterns for service discovery. The only work that addresses service discovery directly was reported by Pärssinen [2004], who introduced a pattern language for service discovery mined from existing discovery protocols. The language describes different aspects of the service discovery process to enable easier comparison of existing approaches.

Elsewhere researchers have posited patterns for service composition. Melloul et al. [2004] expressed high-level patterns as objects that can be specialized to particular applications and reused in the construction of higher-level patterns. Developers write patterns in terms of high-level functions, then decide on the lower-level services to compose. Melloul \& Fox [2004] also discussed reuse trade-offs, showing that too much abstraction makes patterns less expressive. To ensure sufficient expressiveness, all patterns must capture what must be guaranteed in every context of invocation, regardless of the selected services. However this work does not address the automatic discovery or dynamic selection of services. It assumes that developers select services manually at pattern-specialization time.

Closer to our work is that of Tut et al. [2002], who also investigated the use of patterns to facilitate the composition of electronic services. Developers use their domain knowledge to instantiate these patterns, which are indexed using classification codes, to different problem domains. During service composition every task in the pattern is mapped to a service or another pattern. However, again, this work does not address service discovery directly.

Our work is also similar to the use of goal refinement patterns in the KAOS requirements method. Goal refinement and operationalization is poorly supported in many requirements methods, therefore Massonet \& van Lamsweerde [1996] describe the use of patterns to refine and operationalize goals in the problem domain. Patterns in SeCSE are designed to serve a similar purpose - refine a service consumer goal to determine one or more tasks that can be operationalised using invoked services. 


\subsection{Service Discovery in SeCSE}

To ensure industrial uptake, SeCSE's requirements-based service discovery process formulates service queries from use case and requirements specifications expressed in structured natural language. To achieve this, SeCSE's service discovery environment has 4 main components: (i) UCaRE, which supports web-enabled specification of requirements and use cases, and formulation of service queries from these specifications [Jones et al. 2005]: (ii) EDDiE, which uses service queries to discover service descriptions using different retrieval strategies [Zachos \& Maiden 2005]; (iii) SeCSE's service registry - a federated and heterogeneous mechanism for storing service descriptions that is searched by EDDiE; (iv) SeCSE's service explorer environment, which combines text and graphics descriptions of retrieved services to enable selection.

In simple terms, EDDiE uses an analyst-defined service request to generate one or more queries of single or compound natural language terms that it matches to equivalent terms in service descriptions stored in SeCSE's service registries. Distance measures between terms in each query and service description are used to compute the distance between the query and description.

Inevitably, service queries that are derived from incomplete and inconsistent requirements will themselves be incomplete and inconsistent. Therefore EDDiE extends service queries to increase the likelihood of successful service discovery using two core strategies - query expansion and word sense disambiguation - that are implemented in 4 key components shown in Figure 1. In the first the service query is divided into sentences, then tokenized and part-of-speech tagged and modified to include each term's morphological root (e.g. driving to drive, and drivers to driver). In the second the algorithm applies 7 procedures to disambiguate each term by defining its correct sense and tagging it with that sense (e.g. defining a driver to be a vehicle rather than a type of golf club). In the third the algorithm expands each term with other terms that have similar meaning according to the tagged sense, to increase the likelihood of a match with a service description (e.g. the term driver is synonymous with the term motorist which is also then included in the query). In the fourth component the algorithm matches all expanded and sense-tagged query terms to a similar set of terms that describe each candidate service, expressed using the service description facet in the SeCSE service registry. Query matching is in 2 steps: (i) XQuery text-searching functions to discover an initial set of services descriptions that satisfy global search constraints; (ii) traditional vector-space model information retrieval, enhanced with WordNet, to further refine and assess the quality of the candidate service set. This two-step approach overcomes XQuery's limited text-based search capabilities.

The WordNet on-line lexicon fulfills an important role for three of the algorithm's components. WordNet is a lexical database inspired by psycholinguistic theories of human lexical memory [Miller 1993]. It has two important features. Firstly it divides the lexicon into four categories: nouns, verbs, adjectives and adverbs. Word senses for each category are organized into synonym sets (synsets) that represent concepts, and each synset is followed by its definition or gloss that contains a defining phrase, an optional comment and one or more examples. Secondly, WordNet is structured using semantic relations between word meanings that link concepts. Relationships between 
conceptions such as hypernym and hyponym relations are represented as semantic pointers between related concepts [Miller 1993]. A hypernym is a generic term used to designate a whole class of specific instances. For example, vehicle denotes all the things that are separately denoted by the words train, chariot, dogsled, airplane, and automobile, and is therefore a hypernym of each of those words. On the other hand, a hyponym is a specific term used to designate a member of a class, e.g. chauffeur, taxidriver and motorist are all hyponyms of driver. A semantic relation between word meanings, such as a hypernymy, links concepts.

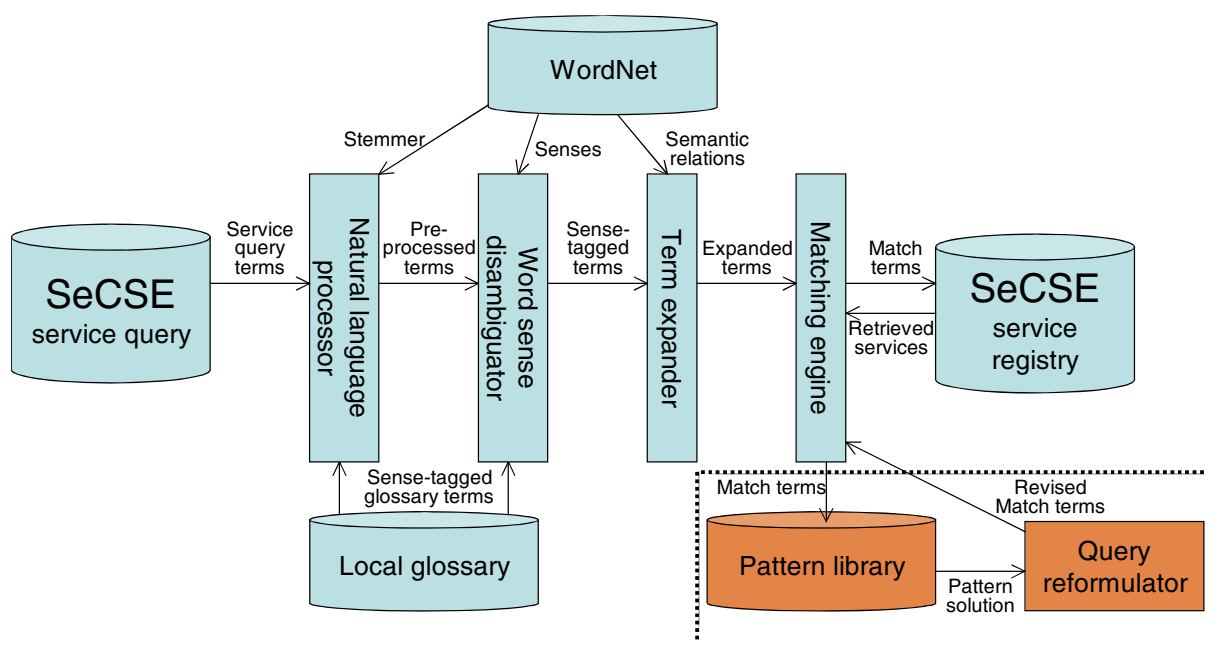

Fig. 1. SeCSE's service discovery algorithm, showing original EDDiE components and our pattern extension to the algorithm below the dotted line

EDDiE implements the WordNet.Net library, the .Net Framework library for WordNet [Crowe 2005]. The library provides public classes that can be accessed through public interfaces. It uses the synonym sets and associated word senses to disambiguate between different meanings of the same word, and semantic relations such hypernym and hyponym as well as synonyms to expand service queries.

Although we treat query expansion and word sense disambiguation as necessary techniques for effective service discovery, we do not believe that, on their own, they are sufficient to overcome the ontological mismatch that will exist between requirement specifications and service descriptions. To deliver this sufficient algorithm, we have developed the pattern extension to EDDiE.

\subsection{Pattern-Based Extension to Service Discovery}

As Figure 1 shows we implement the pattern-based extension by adding a patterns catalogue and a new component - the query reformulator - to EDDiE. Input to this extension is an expanded and disambiguated set of terms in a service query, and output is one or more new service queries reformulated using retrieved patterns. 
Each pattern in the pattern language includes a structured natural language description of a class of problem in context and a structured natural language description of one or more candidate classes of service that are proven solutions to the class of problem. Pattern-based service discovery is in 3 stages:

1. Pattern match: EDDiE uses its existing algorithm [Zachos \& Maiden 2005] to match the expanded and disambiguated service query to the problem description of each pattern. The result is an ordered set of retrieved patterns that match to the service query;

2. Reformulate service request: EDDiE uses the described classes of service in the solution part of each retrieved pattern to generate one or more new service queries that are expressed in terms of the service features rather than consumer requirements;

3. Service match: EDDiE applies each new service query to discover service descriptions from the SeCSE service registries. The result is an ordered set of service descriptions that match the reformulated service query.

We have designed each pattern to enable more effective pattern matching in stage 1 using two WordNet structures. Firstly, each term in the pattern's problem description is pre-tagged with the correct word sense (e.g. a vehicle is a conveyance that transports people or objects), so that EDDiE only applies word sense disambiguation to terms in the service query. Secondly, terms in the problem description are made more generic using hypernym relationships specified in WordNet, thus ensuring that the pattern can be applied more widely in the automotive domain whilst still enabling effective term matching using the hypernym relationship.

Previously we proposed this pattern-based extension to service discovery [Zhu et al. 2005], however both its feasibility and effectiveness were unproven. In the remainder of this paper we report a first evaluation that sought to establish whether: (i) a pattern language could be elicited from domain experts - in our case automotive engineers, and; (ii) the language has the potential to improve service discovery. This paper reports an empirical answer to the first question and an example-based demonstration to begin to answer the second.

\section{Developing a Pattern Language for Vehicle Fleet Management in DaimlerChrysler}

We worked with SeCSE partners DaimlerChrysler to develop and evaluate a prototype pattern language for their on-board systems for fleet vehicle management. Fleet vehicle management enables DaimlerChrysler to maintain effective customer support after sale or lease, to ensure vehicle quality and support vehicle use. The result was a 40-page specification of a language that contained 7 core patterns. Each pattern was described using 4 facets. The first described background information about the pattern in text form and $i^{*}$ strategic dependency (SD) and strategic rationale (SR) models [Yu \& Mylopoulos 1994] that represent and communicate the essence of each pattern. The SD model depicts dependencies between strategic actors whilst the SR model depicts how actors achieve strategic goals in terms of tasks and resources 
[Yu \& Mylopoulos 1994]. We had successfully used $i^{*}$ models to elicit, evaluate and communicate patterns on submarine design with BAE SYSTEMS engineers [Pavan et al. 2003], and applied this method again in SeCSE. The second facet described the pattern's problem in context using structured natural language descriptions in use case specifications [Jacobson et al. 2000] and VOLERE requirement statements [Robertson \& Robertson 1999]. The third facet described the pattern solution in terms of the behaviour of the service-centric application, again as a use case specification. The fourth facet described classes of service implemented in this application, in terms of structured service descriptions from which EDDiE generates revised service queries [Sawyer et al. 2005].

\subsection{Elicitation Method}

Elicitation was in three phases. Each focused on a one-day workshop with DaimlerChrysler engineers in Stuttgart. The engineers were experienced system designers from DaimlerChrysler's Research and Development department, and at least 2 engineers participated in each workshop. Throughout each workshop we encouraged the engineers to converse with each other. This technique, known as constructive interaction [Miyake 1986], overcomes the unnatural aspects of other elicitation techniques. All conversation took place in German, the engineer's native language, and the SeCSE researcher was fluent in the language. All results were then translated into English, the language of the project.

The 3 workshops had 3 different goals:

1. Pattern discovery, to walk through scenarios that envision how vehicle fleet management might take place, and discover and document design decisions about possible on-board service-centric applications. Results provided an outline pattern language and template for each pattern;

2. Pattern definition, to develop and specify complete patterns around the reported design decisions, including the description of possible classes of service that an application might discover and bind to during deployment;

3. Pattern evaluation, to evaluate the specified patterns and revise them in light of feedback.

During the first workshop we walked through DaimlerChrysler scenarios that described how fleet vehicle management took place. Engineers selected the scenarios according to business importance, how frequently these scenarios might occur, and the potential use for future software services. They then elaborated these scenarios into sequences of actions that were walked through to discover design decisions that would need to be made to implement the software services. Next we combined brainstorming with laddering, a form of structured interviewing, to elicit knowledge about different candidate architectures, why each was chosen or rejected, and conditions for the use of each. Laddering questions gathered important data about trade-offs between requirements and the feasibility of different architectures. All data was recorded on flipchart sheets. At the end of the workshop we structured knowledge about each pattern in the background facet. We also developed an $i^{*}$ 
Strategic Dependency (SD) model of the system actors and dependencies described in the pattern.

Prior to the second workshop - pattern definition - we sent each pattern description from the first workshop to the DaimlerChrysler engineers for comment and correction. During the workshop itself we worked with the engineers to develop the $i *$ SR models, gathering data to complete one model for each pattern using brainstorming to discover concepts and laddering to decompose soft goal concepts. We used the $i *$ SR models to decompose modeled tasks into sub-tasks that could be undertaken by remote software services. After the workshop we elaborated the $i^{*}$ models to generate the other 3 facets of each pattern: (i) the problem facet, expressed as classes of behaviour and requirements expressed in a use case specification; (ii) the solution facet, expressed as the use case extended with descriptions of classes of behaviour specific to service-centric applications; (iii) the service classes facet expressed using SeCSE's faceted service specification structure [Sawyer et al. 2005], that was the basis for generating new service queries.

In the third workshop - pattern evaluation - we formally reviewed then evaluated each pattern with the DaimlerChrysler engineers.

\subsection{Results}

During the first workshop we developed and collected a large number of informal artifacts such the mind maps shown in Figure 2. These artifacts provided the basis for pattern definition in the second workshop. All patterns in the language made reference to onboard vehicle boxes that deliver software services to the driver, software servers that DaimlerChrysler supports to deliver services to vehicles, and service providers from whom services are discovered and deployed. Raw data collected from the 3 workshops is reported in Dourdas [2005].
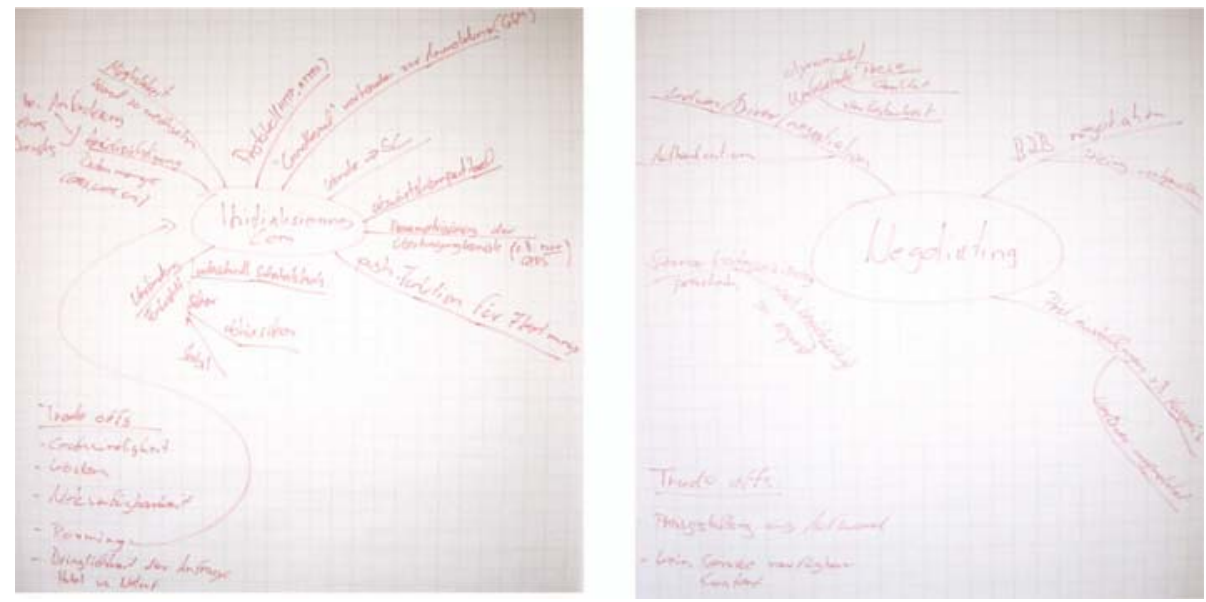

Fig. 2. The mind maps developed for Vehicle box update (on the left) and Negotiation (on the right) 
At the end of the third workshop the language contained 7 core patterns:

1. Communication initialization: Initializing processes prior to data exchange, taking into account requirements from the driver and server;

2. Authentication: Determining identities of actors for legal, billing and security purposes, exploring the trade-offs between user-friendliness and security;

3. Emergency administration: An administrator accessing the vehicle box to recover from malfunctions;

4. Vehicle box update: Updating in response to developer requests and new software versions, taking into account billing and safety requirements;

5. Language selection: Services are offered in different languages chosen by the driver, making use of specialized translation services;

6. Vehicle status sending: Exploiting vehicle-related services (e.g. detection of the next service station) to transmit data such as position and mileage, trading off keeping information current and up-to-date and communication costs;

7. Negotiation: Offering an unlimited amount of service may result in price negotiations with the driver that can lead to usability problems.

Each pattern was described using the background, problem description, solution description and service class facets described earlier. To demonstrate these patterns and the facets, we report the final version of the Negotiation pattern.

\subsection{The Negotiating Pattern}

Background Facet: Drivers use different services from their vehicles, many of which must be paid for. Different service price models and ranges exist, depending on service availability and quality, and the final price must be negotiated by the driver. Pre-defined price preferences speed up the service selection and negotiation but restrict service choice and can lead to driver choice not being met, whilst service providers do not provide transparent and comparable pricing systems. Furthermore, because the driver must accept the price, driver authentication is needed. This leads to a trade-off between the automation of service selection and wider service selection drivers who accept service prices can benefit from more available services, but this requires more interaction.

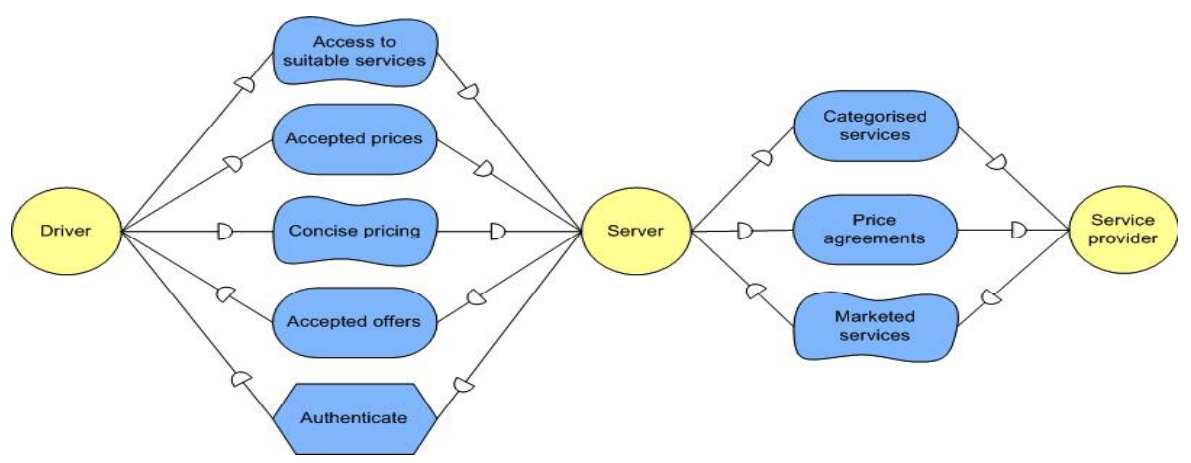

Fig. 3. The $i * \mathrm{SD}$ model for the Negotiating pattern 
The SD model in Figure 3 shows two-way dependencies between the driver and server actors, and between the server and service provider actors. The supporting SR model, not shown due to lack of space, also models driver tasks such as providing authentication and interacting in negotiations, and service provider soft goals such as maximising sale of services.

Problem Facet: EDDiE matches a service query to the use case and requirement specification in Table 1. Specification terms were selected to minimize the number of possible senses, for example the term authentication has just one sense in WordNet. Terms with more than one sense were tagged with WordNet word senses to support query expansion, for example price is tagged as a noun with the sense the amount of money needed to purchase something. Other service-centric computing term senses were specified in the UCaRE lexicon. One example is service, which in WordNet has 15 possible senses as a noun, none of which describe the sense intended in the pattern.

Table 1. Description of the pattern problem for the Negotiation pattern

\begin{tabular}{|l|ll|}
\hline Use case & 1. & The driver requests a service. \\
normal & 2. The server offers a suitable service. \\
course & 3. The driver pays for the service. \\
\hline Requirements & 4. The driver uses the service. \\
\hline & - The driver shall authenticate him/herself before using chargeable services. \\
& - The driver shall be able to accept different prices for services. \\
& - The services shall be categorised into different price groups. \\
& - The driver shall state the price preferences. \\
& - The server shall be responsible for the B2B negotiations with service providers. \\
\hline
\end{tabular}

Solution Facet: Table 2 specifies how the service-centric application will negotiate service prices, in the form of a use case specification. Remote software service actions that EDDiE will need to discover are highlighted in bold. For example, services will be needed to compare service prices and qualities, as specified in action 6.2. However action descriptions on their own are insufficient for discovering solution services, so the fourth facet, service classes, was specified.

Table 2. Description of the pattern solution for the Negotiation pattern

\begin{tabular}{|l|l|}
\hline Use & 1. The driver requests a service. \\
2ase The vehicle box transmits the service request to the server. \\
3. The server requests service information. \\
4. The server retrieves the driver's maximum price preferences. \\
5. The server compares the price preferences with the service price. \\
6. If the service price is higher than the preferred maximum price, \\
6.1. The server searches for alternative services from other service providers. \\
6.2. The server compares the prices, quality and availability of other services. \\
7. If the service price is higher than the preferred maximum price, \\
7.1. The server requests the driver's confirmation. \\
7.2. The driver confirms the higher price. \\
7.3. The server requests the driver's authentication. \\
7.4. The driver enters the driver ID and PIN. \\
7.5. The server validates the authentication data. \\
8. The server invokes the web service.
\end{tabular}


Service Class Facet: EDDiE generates new service queries from service classes of the behaviour depicted in bold in Table 2 to discover concrete services from service registries. Table 3 describes one such class - DriverVehicleAdministration - to implement behaviour specified in use case actions 6.1, 6.2 and 7.5. The SeCSE service discovery algorithm matches attributes of this facet to equivalent attributes of SeCSE's service description facet in service registries [Sawyer et al. 2005]. Other pattern service classes, for example ServiceInformation, are not described in this paper due to lack of space.

Table 3. Description of the DriverVehicleAdministration service class

\begin{tabular}{|c|c|}
\hline Service & DriverVehicleAdministration \\
\hline $\begin{array}{l}\text { Service } \\
\text { Description }\end{array}$ & $\begin{array}{l}\text { The service is able to manage/administrate drivers and vehicles and provides } \\
\text { verification/validation of their identification/authentication details. }\end{array}$ \\
\hline $\begin{array}{l}\text { Service } \\
\text { Goal }\end{array}$ & $\begin{array}{l}\text { Provided with the driver's ID and PIN, the service checks the validity of these } \\
\text { details. In case of new drivers, the service can register them and provide identify- } \\
\text { cation details. The service is also capable of managing vehicles by using their unique } \\
\text { vehicle ID. Linking drivers to certain vehicle enables further levels of authentication. }\end{array}$ \\
\hline $\begin{array}{l}\text { Service } \\
\text { Rationale }\end{array}$ & $\begin{array}{l}\text { In order to avoid many different types of authentication, the service manages drivers } \\
\text { and vehicles for the server, and equally important, for all different service providers. } \\
\text { Otherwise, every single service provider would be responsible for the user } \\
\text { authentication and the drivers would be confronted with different authentication } \\
\text { procedures when using different services. }\end{array}$ \\
\hline $\begin{array}{l}\text { Service } \\
\text { Consumers }\end{array}$ & Server, Administrator, Service Provider \\
\hline $\begin{array}{l}\text { Service } \\
\text { Operations }\end{array}$ & $\begin{array}{l}\text { Validate driver } \\
\text { Validate vehicle } \\
\text { Provide driver identification details } \\
\text { Manage driver - vehicle combinations }\end{array}$ \\
\hline
\end{tabular}

The language's other 6 patterns were structured and described in the same manner [Dourdas 2005]. DaimlerChrysler engineers accepted and signed off the 7 patterns as the basis for a first pattern language with which to evaluate SeCSE's pattern extension to service discovery. The next section demonstrates how service discovery can be enhanced with a simple example that exploits the Negotiation pattern.

\section{Demonstrating Pattern-Based Service Discovery}

In the example requirements analysts use SeCSE's web-based UCaRE tool to specify requirements of service consumers - Mercedes drivers - for an onboard application that provides them with route planning using up-to-date information about traffic conditions. These requirements are expressed as the use cases and VOLERE requirements shown in Figure 4. Each assumes no solution knowledge about journey planner and other services. It is expressed in terms of the problem domain.

EDDiE generates XML service queries from elements in the requirement specification. The query follows an XML schema that defines its structure, content and semantics. EDDiE disambiguates and expands query terms using the components shown in Figure 1 [Zachos \& Maiden 2005]. A fragment of the generated XML query with expanded problem domain terms is shown in Figure 5. The first part lists original query terms with their WordNet word types and senses whilst the second lists 


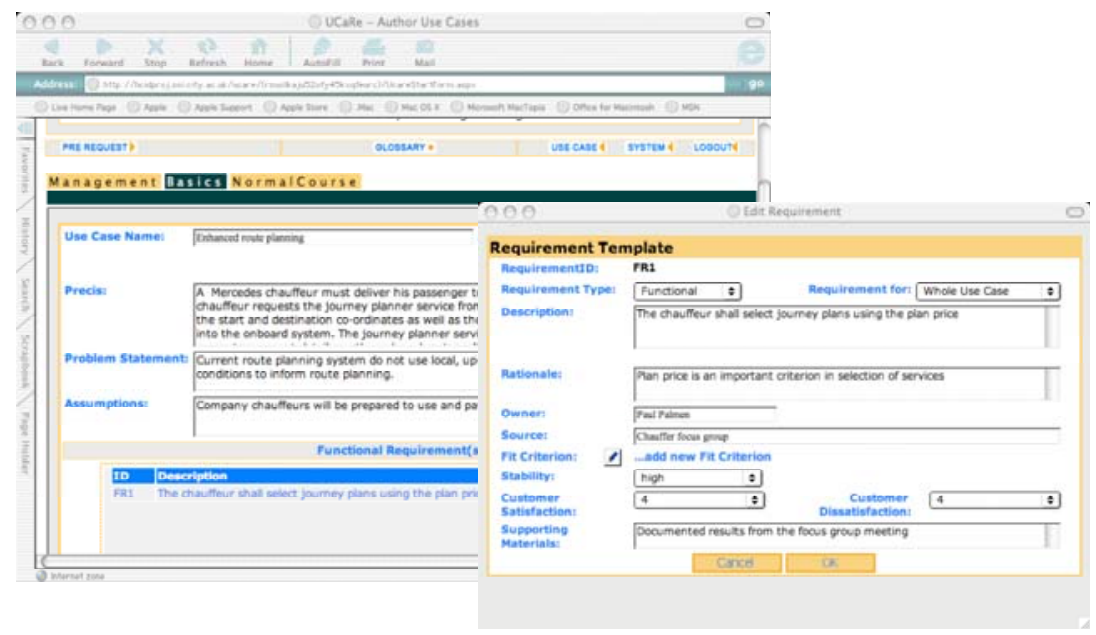

Fig. 4. Use case précis and one requirement for the onboard route planner application, expressed in UCaRE

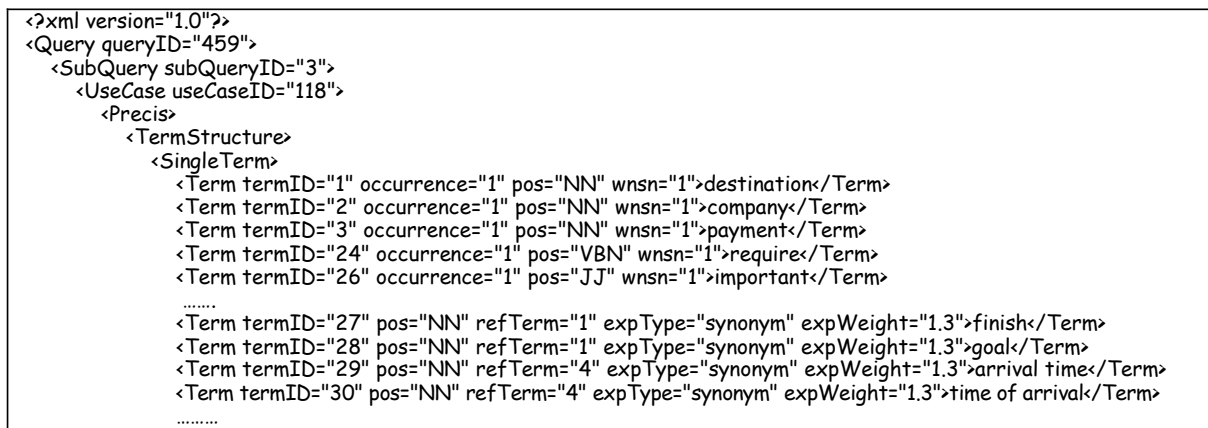

Fig. 5. Part of the generated XML query from the use case in Figure 4, showing original terms and their WordNet word types and word senses (e.g. destination) and expanded terms (e.g. finish and goal) and expansion weightings

expanded terms and expansion weightings. EDDiE matches original and expanded terms to terms that describe pattern problem facets.

SeCSE's pattern catalogue is implemented in eXist, an Open Source native XML database featuring index-based XQuery processing, automatic indexing, and tight integration with XML development tools. EDDiE queries the catalogue using XQuery, a query language designed for processing XML data. XML queries are transformed into one or more XQueries that are fired at the problem description facets of patterns in the catalogue. Figure 6 shows part of the XML describing the Negotiation problem facet. Not shown, again due to lack of space are the tagged word senses (e.g. WordNet sense 1 is tagged to driver - the operator of a motor vehicle), 
whilst other terms are inter-changeable with more generally-applicable WordNet hypernyms, for example request with communicate, and offer with supply.

In our example EDDiE retrieves the Negotiation pattern reported in Section 3 using the generated XQueries. Matched terms include <chauffer, driver $>$, <journey planner, service> and <authenticate, authenticate>. As the match to the Negotiation pattern is greater than a pre-specified match threshold, EDDiE's query reformulator automatically generates new XML queries and XQueries from information in the ServiceInformation and DriverVehicleAdministration service classes specified in the facet. A fragment of the XML query for DriverVehicleAdministration is shown in Figure 7. In contrast with original query terms in Figure 5, terms describe relevant software behaviour to match to terms describing concrete services in registries.

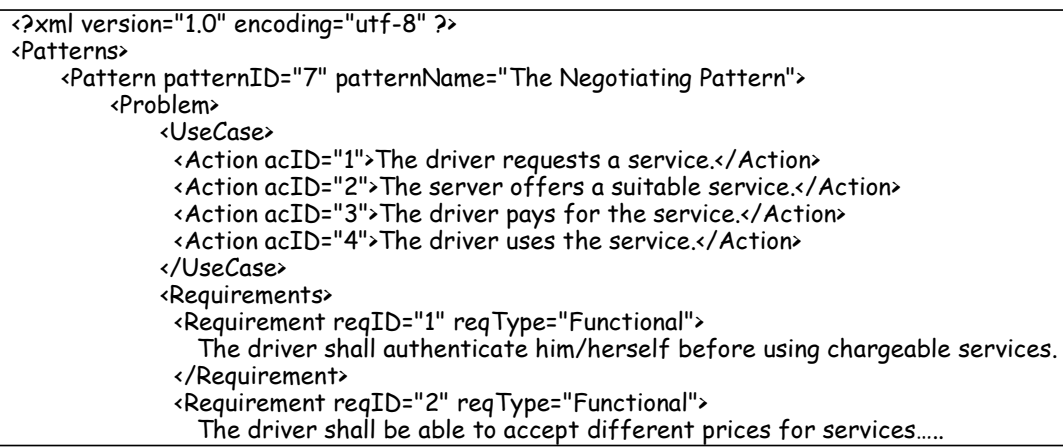

Fig. 6. Partial XML specification of the Negotiation pattern's problem facet

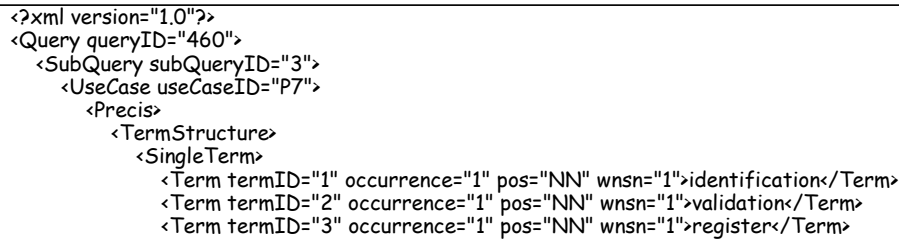

Fig. 7. Part of the reformulated XML query in Figure 5

Next, two things happen. Firstly, UCaRE proposes the pattern solution facet to the requirements analysts to extend the original use case specification shown in Figure 4. Secondly EDDiE fires the reformulated XQuery at the service description facets of services in SeCSE service registries using the existing EDDiE algorithm [Zachos \& Maiden 2005]. The reformulated XQueries include new terms not included in the original query, such as verification, validation, identification, authentication, vehicle identifier, and register. Analysts browse and select between retrieved services using SeCSE's service explorer component. 


\section{Implementation, Discussion and Future Work}

We have implemented the vehicle fleet management pattern catalogue as a local SeCSE service registry without implementations of the specified service classes. Each pattern facet is described using an XML data structure in an eXist database. This implementation is the basis for answering our second question in the future - to explore the utility of pattern-based query reformulation in EDDiE.

Results reported in this paper provided an emphatic answer to the first research question, whether a pattern language for service-centric applications can be elicited. Three workshops, each lasting less than one day with 2 DaimlerChrysler engineers, were sufficient to elicit and describe patterns that elaborate on 7 important servicecentric design decisions. After the third workshop, DaimlerChrysler signed off the language as an accurate representation of domain knowledge about software services for vehicle fleet management. In this regard, these results supported earlier results [Pavan et al. 2003] that support use of a workshop-based elicitation strategy. An initial analysis of the patterns, demonstrated with the reported example, revealed that service classes linked to each problem class have the potential to reformulate EDDiE service queries with new terms indicative of service ontologies. In the future, an evaluation of EDDiE with service queries for vehicle fleet management based on real automotive requirements is planned to determine the relative effectiveness of EDDiE's pattern-based extension. In particular we will explore different pattern configurations, for example the effectiveness of tagging different terms with WordNet senses, and different hypernym terms to use to express the more abstract pattern. We hope to report results in the near future.

Results gave rise to important discussion points. Although the pattern language was developed for remote software services for vehicle fleet management in DaimlerChrysler, some of the patterns have the potential to generalize beyond both the organization and the domain. The reported Negotiation pattern describes classes of candidate service that can reformulate queries in all consumer payment domains including in-car services using more general hypernym relationships, namely driver is-a purchaser. Similar claims can be made for other patterns in the language, for example Authentication, Emergency administration and Language selection. This raises important questions about which levels of abstraction afford best pattern matching and reuse [Sutcliffe \& Maiden 1998]. Melloul \& Fox [2004] report the need for patterns to describe all service invocation contexts, which is neither plausible nor desirable in SeCSE patterns. However deciding what information is needed to describe and discover patterns effectively remains an open question - for example should EDDiE also expand and disambiguate background facet descriptions, and should reformulated queries still include original query terms? During evaluation of EDDiE's pattern-based extension, we will explore these questions with modified pattern versions to other SeCSE domains including telecommunications with other industrial partners Telecom Italia and Telefonica. Finally, the link between the pattern language and emerging standards for semantically enhanced service descriptions such as OWL-S and WSMO warrants some discussion. The language reported in this paper describes DaimlerChrysler expertise using as structured natural language independent of representations needed for implementation in service registries. This is because the design decisions that the engineers make are independent of standards used to 
represent services. During evaluation of the implemented pattern language we will explore whether use of WordNet as a weak ontology or more formal approaches such as WSDL-S improve the representation, discovery and exploitation of patterns.

The related work revealed that our use of patterns in service discovery is unique in service-centric computing. We plan to advance this work by extending patterns with knowledge about service-centric architectures related to each service class that is expressed using UML message sequence charts and OCL constraints describing service operations and query constraints. This will enable more precise behaviourbased service matching [Spanoudakis et al. 2005] directly from problem requirements expression in UCaRE, potentially shortening the time needed to develop servicecentric applications.

\section{Acknowledgements}

The work reported in this paper is funded by the EU Framework VI 511680 SeCSE Integrated Project. We wish to thank all partners for their inputs and feedback so far.

\section{References}

Alexander C., 1979, 'The Timeless Way of Building', NY: Oxford University Press.

Baeza-Yates, R. \& Ribiero-Neto, B., 1999 'Modern Information Retrieval', Addison-Wesley 1999.

Crowe M.,2005, http://opensource.ebswift.com/WordNet.Net

Dourdas N., 2005, 'A Pattern Language for Vehicle Fleet Management in DaimlerChrysler', MSc Thesis, Department Information Science, City University, London, September, 2005.

Gamma E., Helm R., Johnson R. \& Vlissides J., 1995, 'Design Patterns - Elements of Reusable Object-Oriented Software', Addison-Wesley.

Jackson M., 1995, 'Software Requirements and Specifications', Addison-Wesley.

Jacobson I., Booch G. \& Rumbaugh J., 2000, The Unified Software Development Process, Addison-Wesley-Longman.

Jones S.V., Maiden N.A.M., Zachos K. \& Zhu X., 2005, 'How Service-Centric Systems Change the Requirements Process', Proceedings REFSQ'2005 Workshop, in conjunction with CaiSE'2005, 13-14 2005, Porto, Portugal.

Leavitt N., 2004, 'Are Web Services Ready to Deliver?', IEEE Computer, 37(11), 14-18.

Massonet, P. \& van Lamsweerde, A., 1996, 'Formal refinement patterns for goal-driven requirements elaboration', Proceedings of FSE-4 - 4th ACM Symposium on the Foundations of Software Engineering, San Fransisco, ACM Press (1996), 179-190.

Melloul L., Fox A. "Reusable Functional Composition Patterns for Web Services", Second International Conference on Web Services (ICWS04), San Diego, CA, July 6-9, 2004.

Miller K., 1993, 'Introduction to WordNet: an On-line Lexical Database' Distributed with the WordNet software

Mijake N., 1986, 'Constructive Interaction and the Iterative Process of Understanding', Cognitive Science 10, 151-177.

Pärssinen J, Koponen, T. and Eronen P. "Pattern Language for Service Discovery", In Proceedings of the 9th European Conference on Pattern Languages of Programs (EuroPLoP '04), 645-660, Irsee, Germany, July 2004. 
Pavan P., Maiden N.A.M. \& Zhu X., 2003, 'Towards a Systems Engineering Pattern Language: Applying i* to Model Requirements-Architecture Patterns', STRAW'2003, 2nd International Software Requirements and Architectures Workshop, ICSE'2003, May 2003.

Robertson S. \& Robertson J., 1999, Mastering the Requirements Process, Addison-Wesley

Sawyer P., Hutchinson J., Walkerdine J. \& Sommerville I., 2005, 'Faceted Service Specification', Proceedings SOCCER (Service-Oriented Computing: Consequences for Engineering Requirements) Workshop, at RE'05 Conference, Paris, August 2005.

Schmid K., Eisenbarth M. \& Grund M., 2005, 'From Requirements Engineering to Knowledge Engineering: Challenges in Adaptive Systems', Proceedings SOCCER (Service-Oriented Computing: Consequences for Engineering Requirements) Workshop, at RE'05 Conference, Paris, August 2005.

Schütze H. and Pedersen, J.O., 1995 “Information retrieval based on word senses”, in Proceedings of the Symposium on Document Analysis and Information Retrieval, 4: 161- 175, 1995.

Spanoudakis G., Zisman A., Kozlenkov A.: 2005, 'A Service Discovery Framework for Service Centric Systems', to appear in IEEE International Conference on Services Computing, IEEE Computer Society Press, 251-259.

Sutcliffe A.G. \& Maiden N.A.M., 1998, 'The Domain Theory for Requirements Engineering', IEEE Transactions on Software Engineering, 24(3), 174-196.

Tut M. T. \& Edmond, D. 2002 "The Use of Patterns in Service Composition”, Revised Papers International Workshop on Web Services, E-Business, and Semantic Web, 28-40, 2002

Yu E. \& Mylopoulos J.M., 1994, 'Understanding "Why" in Software Process Modelling, Analysis and Design', Proceedings, 16th International Conference on Software Engineering, IEEE Computer Society Press, 159-168.

Zachos K. \& Maiden N.A.M., 2006, 'Discovering Services During Requirements Processes', Technical Report, Centre for HCI Design, City University London.

Zhu H., Maiden N.A.M., Jones S.V., Zachos K., 2005, "Applying Patterns in Service Discovery", Proceedings SOCCER (Service-Oriented Computing: Consequences for Engineering Requirements) Workshop, at RE'05 Conference, Paris, August 2005. 